\title{
The effect of Pressure- or Volume- controlled ventilation on inflammatory factors of patients undergoing surgery with the novel coronavirus (COVID-19) pneumonia: protocol for a systematic review and meta-analysis
}

\section{Bo Jiao}

Sichuan University West China Hospital

Mingyuan Chen

Sichuan University West China Second University Hospital

Xi Luo

Sichuan University West China Hospital

Chan Chen ( $\nabla$ xychenchan@gmail.com )

Sichuan University West China Hospital https://orcid.org/0000-0001-5823-8247

Jin LIU

Sichuan University West China Hospital

Protocol

Keywords: COVID-19, surgery, pressure-controlled ventilation, volume-controlled ventilation, inflammation, ARDS

Posted Date: October 21st, 2021

DOI: https://doi.org/10.21203/rs.3.rs-951429/v1

License: @ (i) This work is licensed under a Creative Commons Attribution 4.0 International License.

Read Full License 


\section{Abstract}

Objective During the COVID-19 pandemic, some patients with novel coronavirus pneumonia may have concurrent disease that must be accepted surgery. However, the extensive inflammatory response existed in this population, especially in the lungs. And various ventilation could affect the inflammation of lungs. Therefore, how to choose the ventilation mode to reduce the effect of mechanical ventilation on inflammation is an important clinical practice. We aim to investigate the effect of different ventilation modes on inflammatory factor levels of patients undergoing surgery with COVID-19 through this systematic review and meta-analysis.

Methods and analysis This systematic review and meta-analysis have been registered in the International Prospective Register of Systematic Reviews (PROSPERO). The PubMed, Embase via Ovid, Chinese Biological Medical Database (CBM), China National Knowledge Infrastructure (CNKI), the Cochrane Central Register of Controlled Trials (CENTRAL) will be searched from inception to date to find relevant studies. In addition, the relevant literature was also hand-searched. Two searchers independently selected the studies, extracted data and assessed the quality of studies included. The Review Manager 5.4 software (The Cochrane library,Oxford,England) will be used for data synthesis and meta-analysis. Continuous variables were embodied by Weight Mean Differences with 95\% confidence intervals $(\mathrm{Cl})$. Dichotomous outcomes were embodied by Odds ratio with $95 \% \mathrm{Cl}$.

Ethics and dissemination Ethical approval is not required because the data was collected from the published studies. Finally, the results of this systematic review will be published in a peer-reviewed journal.

\section{PROSPERO registration number CRD42021251937}

\section{Introduction}

From the end of December 2019 to now, more and more patients have been diagnosed with novel coronavirus (COVID-19) pneumonia, and this epidemic was not limited in the place, Wuhan, China, and caused a global pandemic[1, 2]. Till November 2020, there were more than 8 million cases in India, and it has become one of the countries which had the fastest increasing speed of COVID-19 infection for the terrible prevention and control strategy $[3,4]$. Therefore, it is a challenge for all countries, especially the poor, to manage and control the COVID-19 infection, because of the shortage of medical resources[5].

Patients with novel coronavirus pneumonia would present various clinical manifestations such as fever, cough, dyspnea, and pain, et. al[6]. And in this population, they had an apparent inflammatory reaction. For example, IL-1 $\beta$, IL-6, IL-8 was high, but the IL-10 is low[7]. In patients who suffered from acute respiratory distress syndrome, inflammation seriously increases the injury of different organs, especially the lungs [8]. The COVID-19 infection was a massive hit to people's health, and simultaneously these patients might also suffer from another acute disease that needs to be treated by surgery, such as acute surgical abdomen, and it could be a considerable challenge to manage[9]. In the perioperative period, 
anesthesiologists were among the most critical roles to manage patients undergoing surgery, and the management of the respiratory is the most critical step[10]. The previous studies had found that the mode of ventilation would affect the concentration of inflammatory factors, and pressure-control ventilation might decrease the level of tumor necrosis factor (TNF)- $a$ and interleukin (IL)- 6 and also increase the level of IL-10 compared with volume-controlled ventilation[11]. However, another study revealed no difference in inflammatory factor levels between volume- and pressure-controlled ventilation. So, it is unclear whether the mode of ventilation could influence the level of inflammatory marker. However, these factor levels are important for surgical patients' recovery, especially the persons with novel coronavirus pneumonia who have a high level of inflammation.

Therefore, to the anesthesiologists' best to minimize the influence of anesthesiology on the inflammatory level is significantly essential. So, it is significant to investigate the effect of various ventilation modes on inflammatory factors of surgical patients with COVID-19. Additionally, till now, no systematic review and meta-analysis are examining the influence of the ventilation approach on inflammatory marker levels of patients undergoing surgery with COVID-19. Therefore, we will qualitatively and quantitatively investigate the effects of pressure- or volume-controlled ventilation on patients' inflammatory factors with novel coronavirus pneumonia in this systematic review and meta-analysis.

\section{Methods}

This protocol of systematic review and meta-analysis was conducted in line with the Preferred Reporting Items for Systematic Reviews and Meta-analyses Protocols (the PRISMA-P) [12]. The PROSPERO registration number is CRD42021251937. For the nature of this article, no informed consent of patients was needed.

\section{Literature search}

The PubMed, Web of Science, Embase via Ovid, Chinese Biological Medical Database(CBM), China National Knowledge Infrastructure(CNKI), the Cochrane Central Register of Controlled Trials (CENTRAL) will be searched by two reviewers independently to full access to relevant research from inception to date. In addition, the relevant pieces of literature were also hand-searched. Studies concerning the effects of ventilation mode for patients' inflammatory factor levels with COVID-19 in the surgery period will be brought into this meta-analysis, including randomized controlled trials (RCTs), controlled clinical trials (CCTs), prospective and retrospective comparative cohort studies, cross-sectional researches and observational researches. The search terms included 'pressure-controlled ventilation' and 'volumecontrolled ventilation' and 'COVID-19' or 'novel coronavirus pneumonia' or 'SARS-CoV-2', and 'inflammation', and the detailed search strategy of PubMed is presented in Table 1. There are no language restrictions.

\section{Table 1: search strategy of PubMed}




\begin{tabular}{|c|c|}
\hline Search & Query \\
\hline$\# 1$ & "covid 19"[MeSH Terms] \\
\hline $\begin{array}{l}\# 2 \\
\text { "covid } 1 \\
\text { [Supple" } \\
\text { testing" } \\
\text { [MeSH } \\
\text { Fields] } \\
\text { OR "nco } \\
\text { Fields] } \\
\text { Terms] } \\
\text { OR "cov } \\
\text { "covid 1 } \\
\text { [All Fielc } \\
\text { Fields] } \\
\text { Fields] } \\
\text { [All Fielc } \\
\text { OR "cov } \\
\text { disease } \\
\text { infectio } \\
\text { Fields]) }\end{array}$ & 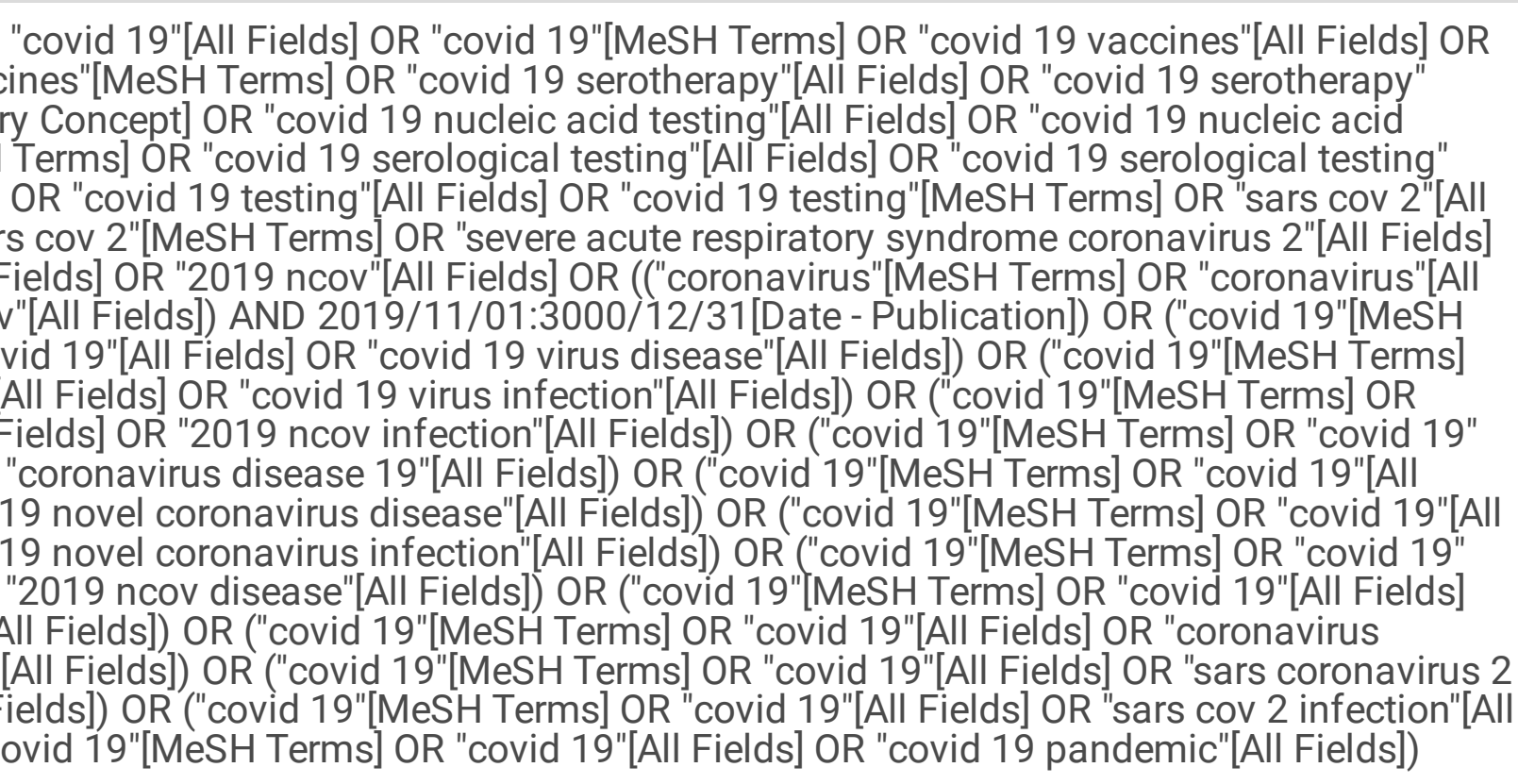 \\
\hline \#3 & \#1 or \#2 \\
\hline $\begin{array}{l}\# 4 \\
\text { OR "ven } \\
\text { Fields] } \\
\text { Terms] } \\
\text { Fields] }\end{array}$ & $\begin{array}{l}\text { "pressure-controlled"[All Fields] AND ("ventilated"[All Fields] OR "ventilates"[All Fields] } \\
\text { g"[All Fields] OR "ventilation"[MeSH Terms] OR "ventilation"[All Fields] OR "ventilate"[All } \\
\text { ntilations"[All Fields] OR "ventilator s"[All Fields] OR "ventilators, mechanical"[MeSH } \\
\text { entilators"[All Fields] AND "mechanical"[All Fields]) OR "mechanical ventilators"[All } \\
\text { ntilator"[All Fields] OR "ventilators"[All Fields] OR "ventillation"[All Fields]) }\end{array}$ \\
\hline $\begin{array}{l}\# 5 \\
\text { OR "ven } \\
\text { Fields] } \\
\text { Terms] } \\
\text { Fields] }\end{array}$ & $\begin{array}{l}\text { "volume-controlled"[All Fields] AND ("ventilated"[All Fields] OR "ventilates"[All Fields] } \\
\text { "[All Fields] OR "ventilation"[MeSH Terms] OR "ventilation"[All Fields] OR "ventilate"[All } \\
\text { tiilations"[All Fields] OR "ventilator s"[All Fields] OR "ventilators, mechanical"[MeSH } \\
\text { entilators"[All Fields] AND "mechanical"[All Fields]) OR "mechanical ventilators"[All } \\
\text { ntilator"[All Fields] OR "ventilators"[All Fields] OR "ventillation"[All Fields]) }\end{array}$ \\
\hline \#6 & \#4 or \#5 \\
\hline $\begin{array}{l}\# 7 \\
\text { Fields] } \\
\text { AND ("re } \\
\text { [All Fielc } \\
\text { OR "resp }\end{array}$ & $\begin{array}{l}\text { "inflammation"[MeSH Terms] OR "inflammation"[All Fields] OR "inflammations"[All } \\
\text { lammation s"[All Fields] OR ("inflammatories"[All Fields] OR "inflammatory"[All Fields]) } \\
\text { e"[All Fields] OR "responses"[All Fields] OR "responsive"[All Fields] OR "responsiveness" } \\
\text { "responsivenesses"[All Fields] OR "responsives"[All Fields] OR "responsivities"[All Fields] }\end{array}$ \\
\hline \#8 & $\# 3$ and \#6 and \#7 \\
\hline
\end{tabular}

\section{Eligibility Criteria}

\section{Type of study design}

All relevant studies, such as RCTs, CCTs, prospective and retrospective comparative cohort studies, crosssectional researches, and observational researches, will be covered in this meta-analysis. Due to the deficiency of original trials, we try our best to find out as many relevant studies as possible. 


\section{Participants}

The patients with COVID-19 who have been controlled breathing through pressure- or volume-controlled mode in the surgery will be involved. Because the number of concerning studies is relatively small, we do not restrict the population's age. Adult and pediatric patients will be included in this meta-analysis. The studies from various countries will be included in the world.

\section{Interventions}

The interventions will be various ventilation modes, including Pressure-controlled ventilation and Volumecontrolled ventilation during the surgery period.

\section{Outcomes}

The primary outcome is the inflammatory factor levels in $3 \mathrm{~h}, 6 \mathrm{~h}, 12 \mathrm{~h}$, and $24 \mathrm{~h}$ after surgery, including IL-6, IL-8, TNF-a, and IL-10. And the secondary outcomes are as follows: postoperative oxygenation index, blood oxygen saturation, serum creatinine concentration, incision infection rate, and hospitalization time.

\section{Study Selection And Data Collection}

Two searchers will independently select the studies. If some discrepancies exist between the searchers, further discussion will be done with a senior reviewer to solve this problem. After removing the duplicates, we will assess the preliminary quality of studies according to the title and abstract. And then, we will do the final assessment by reading the full text. The selection flow gram is presented in Fig. 1. A standard collection form will be adopted to extract the data by two reviewers independently. Any discrepancies will be solved by another senior reviewer. The extracted data including first author, date of publication, sample size, patient characteristics, ASA physical status, type of surgery, type of study, ventilation mode, surgery and anesthesia time, postoperative laboratory results, inhaled oxygen concentration after surgery, patients' number of incision infection, and length of hospitalization. For the missing data, we will contact the author to get it.

\section{Risk-of-bias Assessment And Quality Assessed}

Two tools would be used to evaluate the quality of studies included. The randomized controlled trials were evaluated by Cochrane's risk-of-bias tool according to the following domains: bias arising from the randomization process, bias due to deviations from intended interventions,bias owing to missing outcome data, bias from the measurement of the outcome, bias from the selection of the reported result[13]. And for the non-randomized studies, we will use the Newcastle-Ottawa Scale (NOS) to evaluate the bias, including subjects selection, study comparability, and outcome measurement [14]. According to the tool of assessing the risk of bias, the decisions could be 'Low', or 'High' risk of bias, or express 'Some concerns'. The risk of bias would be assessed by two searchers independently. And the quality of 
evidence about the studies included was evaluated with the Grading of Recommendations, Assessment, Development, and Evaluation (GRADE) criteria[15].

\section{Data synthesis and statistical analysis}

The Review Manager 5.4 software (The Cochrane library, Oxford, England) was used for data synthesis and meta-analysis. Continuous and dichotomous outcomes were embodied by Weight Mean Differences [WMD, 95\% confidence intervals $(\mathrm{Cl})$ ] and odds ratios [OR, 95\% $\mathrm{Cl}$ ], respectively. The heterogeneity would be tested with the $\mathrm{I}^{2}$ index $\left(\mathrm{I}^{2}>50 \%\right.$ indicated significant heterogeneity) [16]. If data had significant heterogeneity, a random effect model would be used. Without obvious heterogeneity, a fixed-effect model would be applied.

\section{Subgroup analysis, sensitivity analysis, and publication bias assessment}

If significant heterogeneity existed, subgroup analysis would be done depending on the

severity of COVID-19 and surgery type. Subgroup analysis was executed when there were at least two studies in each subgroup. Sensitivity analysis was performed by repetitively taking away one study at one time. If the final pooled results did not change after removing one study, the meta-analysis would be considered to be reliable and stable[17]. Funnel plot asymmetry was used to detect publication bias[18].

\section{Ethics And Dissemination}

Ethical approval is not required for the reason that the data was collected from the published reports. Finally, the results of this systematic review will be published in a peer-reviewed journal.

\section{Discussion}

As far as we know, this is the first meta-analysis to examine the effects of Pressure- or Volume-controlled ventilation on patients' inflammatory factor levels with novel coronavirus pneumonia who have experienced surgery. It is crucial for patients who have undergone surgery with COVID-19 to select a proper ventilation mode during the anesthesia period [19]. In this population, the extensive inflammatory response is a significant feature, especially the location of the lungs, so it is vital to reduce this response by using a fit ventilation method. However, it is uncertain whether ventilation mode could influence the inflammatory marker levels in patients undergoing surgery with coronavirus pneumonia due to limited original searches. This is the significant problem which we concern. And also, there is the main limitation in our meta-analysis for the insufficiency of RCTs, which may reduce the strength of evidence.

\section{Declarations}

\section{Ethics approval and consent to participate}

Not applicable 


\section{Consent for publication}

Not applicable.

\section{Availability of data and materials}

Not applicable.

\section{Competing interests}

The authors declare that they have no competing interests.

\section{Funding}

This review did not receive any specific grant from funding agencies in the public, commercial, or not-forprofit sectors.

\section{Authors' contributions}

BJ and MYC designed this review and made the search strategy. BJ and MYC completed the literature search, data collection. BJ and XL will perform the data synthesis and analysis, and BJ was the major contributor to write the protocol draft writing, $\mathrm{JL}$ and $\mathrm{CC}$ revised the manuscript critically. All authors have read and approved the final version.

\section{Acknowledgements}

Not applicable

\section{References}

1. Salata C, Calistri A, Parolin C, et al: Coronaviruses: a paradigm of new emerging zoonotic diseases. Pathogens and Disease 2019; 77(9).

2. Buhat $\mathrm{CAH}$, Torres $\mathrm{MC}$, Olave $\mathrm{YH}$, et al: A mathematical model of COVID-19 transmission between frontliners and the general public. Network Modeling and Analysis in Health Informatics and Bioinformatics 2021; 10(1).

3. Hotez PJ, Fenwick A, Molyneux D. The new COVID-19 poor and the neglected tropical diseases resurgence. Infectious Diseases of Poverty 2021; 10(1).

4. Dhamodharavadhani S, Rathipriya R. Novel COVID-19 Mortality Rate Prediction (MRP) Model for India Using Regression Model With Optimized Hyperparameter. Journal of Cases on Information Technology. 2021;23(4):1-12.

5. Sun SY, Xie Z, Yu KT, et al: COVID-19 and healthcare system in China: challenges and progression for a sustainable future. Globalization and Health 2021;17(1). 
6. Chen NS, Zhou M, Dong X, et al. Epidemiological and clinical characteristics of 99 cases of 2019 novel coronavirus pneumonia in Wuhan, China: a descriptive study. Lancet. 2020;395(10223):50713.

7. McElvaney OJ, McEvoy NL, McElvaney OF, et al. Characterization of the Inflammatory Response to Severe COVID-19 Illness. Am J Respir Crit Care Med. 2020;202(6):812-21.

8. de la Rica R, Borges M, Gonzalez-Freire M. COVID-19: In the Eye of the Cytokine Storm. Frontiers in immunology. 2020;11:558898.

9. Serban D, Socea B, Badiu CD, et al: Acute surgical abdomen during the COVID-19 pandemic: Clinical and therapeutic challenges. Experimental and Therapeutic Medicine 2021; 21(5).

10. Bigatello L, Pesenti A. Respiratory Physiology for the Anesthesiologist. Anesthesiology. 2019;130(6):1064-77.

11. Tan J, Song Z, Bian Q, et al. Effects of volume-controlled ventilation vs. pressure-controlled ventilation on respiratory function and inflammatory factors in patients undergoing video-assisted thoracoscopic radical resection of pulmonary carcinoma. Journal of thoracic disease. 2018;10(3):1483-9.

12. Moher $D$, Shamseer $L$, Clarke M. et al: Preferred reporting items for systematic review and metaanalysis protocols (PRISMA-P) 2015 statement. Systematic reviews. 2015;4(1):1.

13. Higgins JPT, Altman DG, Gotzsche PC. et al: The Cochrane Collaboration's tool for assessing risk of bias in randomised trials. BMJ-British Medical Journal 2011; 343.

14. Stang A. Critical evaluation of the Newcastle-Ottawa scale for the assessment of the quality of nonrandomized studies in meta-analyses. Eur J Epidemiol. 2010;25(9):603-5.

15. Guyatt GH, Oxman AD, Vist GE. et al: GRADE: an emerging consensus on rating quality of evidence and strength of recommendations. BMJ. 2008;336(7650):924-6.

16. Huedo-Medina TB, Sánchez-Meca J, Marín-Martínez F. et al: Assessing heterogeneity in metaanalysis: Q statistic or 12 index? Psychol Methods. 2006;11(2):193-206.

17. Leeflang MM. Systematic reviews and meta-analyses of diagnostic test accuracy. Clinical microbiology and infection: the official publication of the European Society of Clinical Microbiology and. Infectious Diseases. 2014;20(2):105-13.

18. Egger M, Davey Smith G, Schneider M, et al. Bias in meta-analysis detected by a simple, graphical test. BMJ. 1997;315(7109):629-34.

19. Bagchi A, Rudolph MI, Ng PY, et al. The association of postoperative pulmonary complications in 109,360 patients with pressure-controlled or volume-controlled ventilation. Anaesthesia. 2017;72(11):1334-43.

\section{Figures}



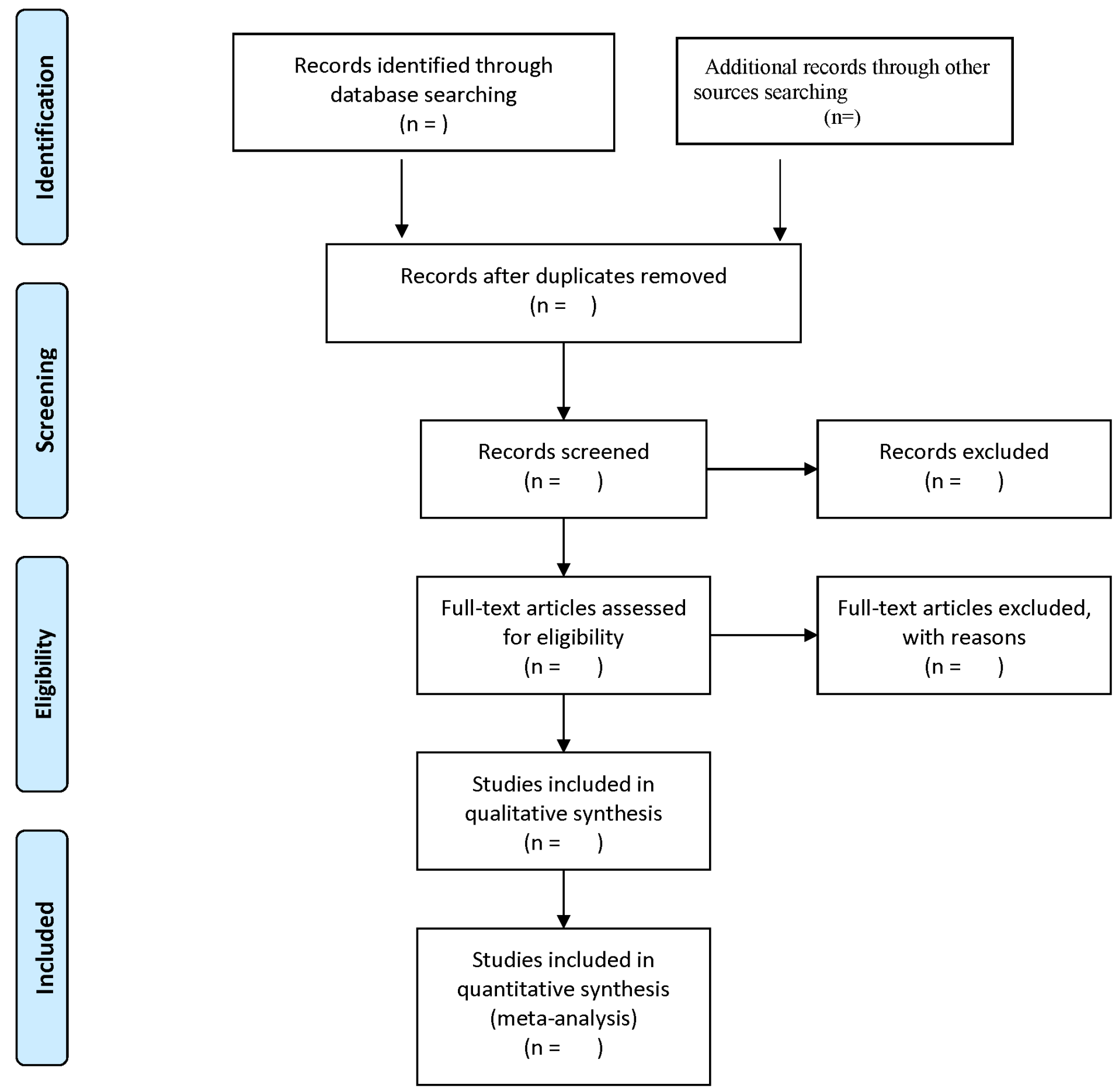

\section{Figure 1}

The flow diagram of literature search and selection

\section{Supplementary Files}

This is a list of supplementary files associated with this preprint. Click to download. 
- PRISMAPchecklist.doc

Page 10/10 